\title{
10-DEBC Hydrochloride as a Promising New Agent against Infection of Mycobacterium abscessus
}

\author{
Da-Gyum Lee ${ }^{1}{ }^{\oplus}$, Hye-Jung Kim ${ }^{2}$, Youngsun Lee ${ }^{3}$, Jung-Hyun Kim ${ }^{3}{ }^{\oplus}$, Yoohyun Hwang ${ }^{1}{ }^{\circledR}$, Jeongyeop Ha ${ }^{2}$ \\ and Sungweon Ryoo $1, * \mathbb{D}$ \\ 1 Center for Clinical Research, Masan National Tuberculosis Hospital, Changwon 51755, Korea; \\ sh050301@naver.com (D.-G.L.); hwangyoohyun@gmail.com (Y.H.) \\ 2 New Drug Development Center, KBIO OSONG Medical Innovation Foundation, Cheongju 28160, Korea; \\ hjkim@kbiohealth.kr (H.-J.K.); myid15@kbiohealth.kr (J.H.) \\ 3 Division of Intractable Diseases Research, Department of Chronic Diseases Convergence Research, \\ Korea National Institute of Health, Cheongju 28160, Korea; iadoj@korea.kr (Y.L.); kjhcorea@korea.kr (J.-H.K.) \\ * Correspondence: viweon@gmail.com; Tel.: +82-55-249-5620; Fax: +82-55-249-5629
}

Citation: Lee, D.-G.; Kim, H.-J.; Lee, Y.; Kim, J.-H.; Hwang, Y.; Ha, J.; Ryoo, S. 10-DEBC Hydrochloride as a Promising New Agent against Infection of Mycobacterium abscessus. Int. J. Mol. Sci. 2022, 23, 591. https:// doi.org/10.3390/ijms23020591

Academic Editor: Maria Rosalia Pasca

Received: 9 November 2021

Accepted: 3 January 2022

Published: 6 January 2022

Publisher's Note: MDPI stays neutral with regard to jurisdictional claims in published maps and institutional affiliations.

Copyright: (C) 2022 by the authors. Licensee MDPI, Basel, Switzerland. This article is an open access article distributed under the terms and conditions of the Creative Commons Attribution (CC BY) license (https:// creativecommons.org/licenses/by/ $4.0 /)$.

\begin{abstract}
Mycobacterium abscessus (M. abscessus) causes chronic pulmonary infections. Its resistance to current antimicrobial drugs makes it the most difficult non-tuberculous mycobacteria (NTM) to treat with a treatment success rate of $45.6 \%$. Therefore, there is a need for new therapeutic agents against $M$. abscessus. We identified 10-DEBC hydrochloride (10-DEBC), a selective AKT inhibitor that exhibits inhibitory activity against $M$. abscessus. To evaluate the potential of 10-DEBC as a treatment for lung disease caused by M. abscessus, we measured its effectiveness in vitro. We established the intracellular activity of 10-DEBC against M. abscessus in human macrophages and human embryonic cell-derived macrophages (iMACs). 10-DEBC significantly inhibited the growth of wild-type M. abscessus and clinical isolates and clarithromycin (CLR)-resistant M. abscessus strains. 10-DEBC's drug efficacy did not have cytotoxicity in the infected macrophages. In addition, 10-DEBC operates under anaerobic conditions without replication as well as in the presence of biofilms. The alternative caseum binding assay is a unique tool for evaluating drug efficacy against slow and nonreplicating bacilli in their native caseum media. In the surrogate caseum, the mean undiluted fraction unbound $(f u)$ for 10 -DEBC is 5.696. The results of an in vitro study on the activity of M. abscessus suggest that 10 -DEBC is a potential new drug for treating M. abscessus infections.
\end{abstract}

Keywords: Mycobacterium abscessus; 10-DEBC; drug resistance; nonreplicating condition; surrogate caseum binding assay

\section{Introduction}

NTM is ubiquitous in soil and drinking water sources [1]. NTM often results in high person-to-person contact rates with pathogens [1,2]. Infections caused by NTM are increasing rapidly, and in developed countries with abundant resources, these infections surpass tuberculosis [3]. Factors contributing to the increased incidence of NTM infection include an increase in susceptible host populations, such as the elderly and those with cellular immune system defects due to disease or immunosuppressive therapy $[3,4]$.

M. abscessus is one of the most common NTMs in pulmonary infections that can cause lung or other diseases in healthy individuals. The number of infections affecting the extrapulmonary part of the body is also increasing [3,5,6].

$M$. abscessus belongs to Runyon group IV, classified as rapid-growing mycobacteria (RGM). It has been reported that RGM in Japan and America causes $5 \%$ of pulmonary infections, and the $65 \%-80 \%$ of the RGM infections are caused by M. abscessus [2]. Pulmonary disease caused by M. abscessus is common in patients with cystic fibrosis (CF), while disseminated infections occur in immunocompromised patients. Patients with CF have an increased risk of disease with M. abscessus [7]. Prospective studies have shown that 
CF is one of the major risk factors for NTM infection, and along with $M$. abscessus is the most common by far [8,9]. In CF, M. abscessus causes rapid inflammatory lung injury [10], often untreatable, and precludes safe lung transplantation, leading to poor clinical outcomes or lifelong asymptomatic infection [11-14].

The alarming fact is that M. abscessus is the most common solitary lung infection, with an average treatment success rate of only $45.6 \%[15,16]$. The specific treatment success rate was $56.7 \%$ for $M$. abscessus subsp. Massiliense [15]. M. abscessus is one of the most resistant microorganisms to chemotherapeutic therapy, and its main threat as a human pathogen is its high resistance to multi-drugs. M. abscessus is also associated with biofilm formation, germicide resistance, high temperatures, and acidic environments [17]. For patients with chronic lung disease due to NTM, none of the currently available treatments have been proven effective for long-term sputum conversion [15]. The high resistant rate of M. abscessus may even disable antibiotic combination therapy.

Current regimens recommend drug combinations for the treatment of $M$. abscessus pulmonary infections. The American Thoracic Society and the Infectious Disease Society of America recommend CLR, amikacin (AMK), azithromycin (AZR), cephalosporin (a type of $\beta$-lactam), cefoxitin (FOX), and carbapenem (another type of $\beta$-lactam) imipenem (IPM). Still, less than half of patients with $M$. abscessus infection can be cured with these treatments $[4,18]$. Consequently, identifying novel treatment approaches is imperative. There is increasing research on experimental antibiotics that have potential activity against $M$. abscessus through various mechanisms and new therapies. However, there is not enough research on its effectiveness against $M$. abscessus.

10-DEBC is a selective Akt inhibitor (also known as PKB) [19]. Akt is a serine/threonine kinase. It phosphorylates and inactivates components of the apoptotic mechanism, including caspase 9 and BCL2-associated agonist of cell death (BAD). Akt phosphorylates and inhibits the forkhead box's transcription factor, thus promoting cell survival [20].

Han et al. identified a novel compound called 10-DEBC [21]. However, the mechanism of $10-\mathrm{DEBC}$ in M. abscessus is not yet well understood. This study demonstrated that 10-DEBC exhibited activity against $M$. abscessus, both in vitro and intracellular.

\section{Results}

\subsection{0-DEBC Is Active against M. abscessus}

The Clinical \& Laboratory Standards Institute (CLSI) currently recommends using the Mueller-Hinton (MH) microdilution broth-based method for determining the MIC of antimicrobial agents for RGM [22]. Therefore, we obtained MIC of 10-DEBC for M. abscessus in $\mathrm{MH}$ broth with resazurin rather than the $7 \mathrm{H} 9$ broth-based method.

The $\mathrm{IC}_{50}$ of $10-\mathrm{DEBC}$ for M. abscessus was $3.006 \mu \mathrm{g} / \mathrm{mL}$ in $\mathrm{MH}$ broth and $5.81 \mu \mathrm{g} / \mathrm{mL}$ in $7 \mathrm{H} 9$ broth, respectively (Figure S1). The $\mathrm{MIC}_{90}$ of 10-DEBC for M. abscessus was $4.766 \mu \mathrm{g} / \mathrm{mL}$ in $\mathrm{MH}$ broth and $9.53 \mu \mathrm{g} / \mathrm{mL}$ in $7 \mathrm{H} 9$ broth, respectively (Figure S1). 10-DEBC exercised similar powers to reference strains of $M$. abscessus, regardless of the type of culture medium $\mathrm{MH}$ or Middlebrook 7H9. As shown in Figure 1A, 10-DEBC showed excellent in vitro activity comparable to other reference compounds, such as CLR, AMK, and streptomycin (STR). They have different mechanisms of action.

In our study, the in vitro $\mathrm{IC}_{50}$ of CLR, AZR, AMK, and STR against M. abscessus was $0.613,5.784,1.34$, and $18.52 \mu \mathrm{g} / \mathrm{mL}$, respectively. The $\mathrm{MIC}_{90}$ of CLR, AZR, AMK, and STR against $M$. abscessus was $1.869,39.251,3.125$, and $36.34 \mu \mathrm{g} / \mathrm{mL}$, respectively.

We have adapted reporter-based assays well suited for drug discovery applications because of their simplicity and sensitivity compared with dye and absorbance-based assays [23]. We constructed luminescence reporter strains and then used them to determine the MICs of CLR and 10-DEBC. First, the in vitro activities of 10-DEBC and CLR were measured by reporter-based bioluminescence; then, MIC data were collected using the REMA method. We used bioluminescent strains of $M$. abscessus to confirm our drug susceptibility. The dose-response curves (DRC) of 10-DEBC in M. abscessus-LuxG13 are 
shown in Figure $1 \mathrm{~B}$, and the $\mathrm{IC}_{50}$ value is $3.9 \mu \mathrm{g} / \mathrm{mL}$ and $\mathrm{MIC}_{90}$ value is $12.5 \mu \mathrm{g} / \mathrm{mL}$. Therefore, 10-DEBC can be considered an effective drug candidate for M. abscessus.
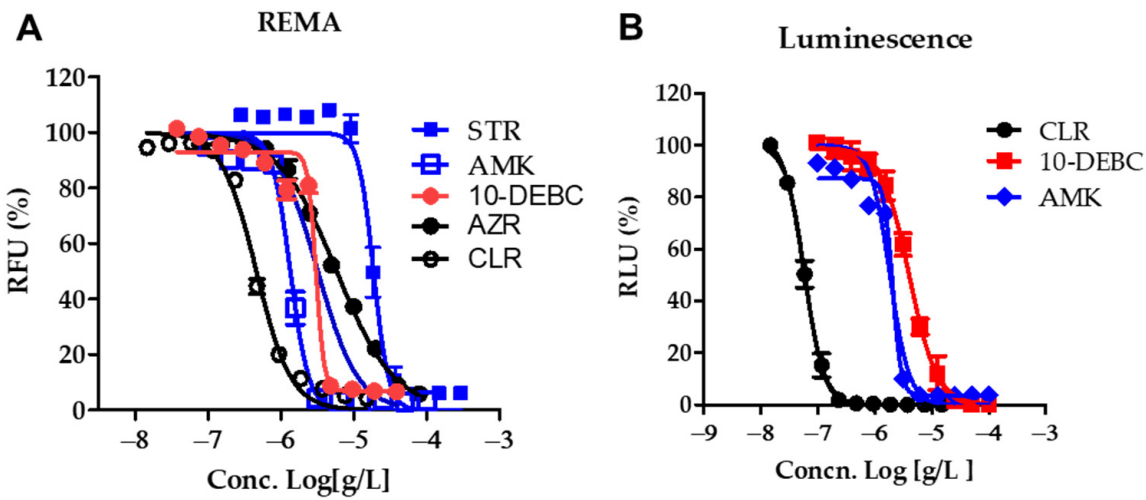

Figure 1. In vitro activity of 10-DEBC. (A) The activity of 10-DEBC against $M$. abscessus in MH broth medium. DRC was plotted from the REMA. RFU, relative fluorescence units. (B) The activity of 10-DEBC against $M$. abscessus-Lux, incubated under the same condition as in panel B RLU, relative luciferase units. The experiments were carried out with three biological replicates and expressed as the mean \pm SEM for each concentration.

\subsection{0-DEBC Is Active against Clinical Isolates of the M. abscessus and Clarithromycin Resistant Mutant}

We next determined whether 10-DEBC retained this potent activity compared with clinical isolates of M. abscessus, including Rough (R) and Smooth (S) colony types. 10-DEBC was also inhibited against nine strains in the clinical isolates, with $\mathrm{IC}_{50}$ from 1.69 to $2.63 \mu \mathrm{g} / \mathrm{mL}$ and $\mathrm{MIC}_{90}$ from 2.38 to $4.77 \mu \mathrm{g} / \mathrm{mL}$, similar to the reference strains of the subspecies (Table 1). The $\mathrm{R}$ phenotype tends to be much stronger than the $S$ type in combating the host defense mechanism $[24,25]$. These results indicate that 10-DEBC is effective in vitro against the reference strain and clinically isolated $\mathrm{R}$ and $\mathrm{S}$.

CLR is a key agent in the treatment of $M$. abscessus complex infections. The main cause of treatment failure is clarithromycin inducible resistance [26]. From this study, we tested whether 10-DEBC effectively inhibited the growth of drug-resistant strains that were laboratory generated from this study at high concentrations (100 Please consider this suggested change and same as follows.) of CLR. As shown in Figure 2, the laboratorygenerated resistance mutant showed high resistance levels to the CLR.

CLR-R M. abcessus
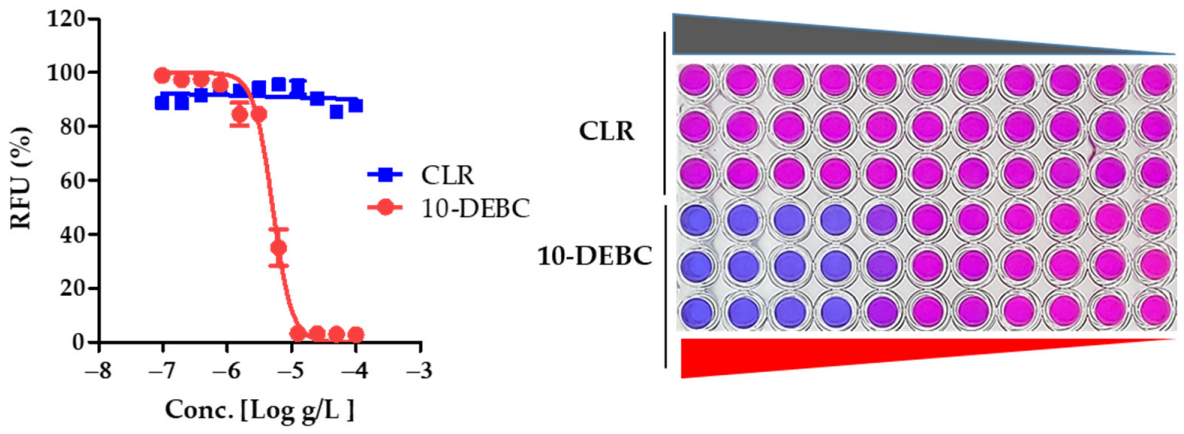

Figure 2. The activity of 10-DEBC against clarithromycin-resistant $M$. abscessus mutants. Clarithromycin-resistant M. abscessus $100 \mu \mathrm{g} / \mathrm{mL}$ to $97 \mu \mathrm{g} / \mathrm{mL}$ of CLR and 10-DEBC. DRC of M. abscessus CLR-R mutant (Left panel). Resazurin reports bacterial viability via a color change from blue to pink (Right panel). Upon oxidation by live M. abscessus, it turns pink, indicating growth of M. abscessus. Each experiment was performed in triplicate. This result was made from a representative experiment. The experiments were carried out with three biological replicates and expressed as the mean \pm SEM for each concentration. 
Table 1. The activity of 10-DEBC and antimicrobial agents against M. abscessus clinical isolates.

\begin{tabular}{|c|c|c|c|c|c|c|c|c|c|c|}
\hline No. & Clinical Isolates & $\begin{array}{c}\text { 10-DEBC } \\
\left(\mathrm{IC}_{50}\right)\end{array}$ & $\begin{array}{l}\text { AMK } \\
\text { (MIC) }\end{array}$ & $\begin{array}{c}\text { CLR } \\
\text { (MIC) }\end{array}$ & $\begin{array}{c}\text { FOX } \\
(\mathrm{MIC})\end{array}$ & $\begin{array}{l}\text { Doxycycline } \\
\text { (MIC) }\end{array}$ & $\begin{array}{l}\text { Linezolid } \\
\text { (MIC) }\end{array}$ & $\begin{array}{c}\text { IPM } \\
\text { (MIC) }\end{array}$ & $\begin{array}{l}\text { Moxifloxacin } \\
\text { (MIC) }\end{array}$ & $\begin{array}{c}\text { Trimethoprim/ } \\
\text { Sulfamethoxazole } \\
\text { (MIC) }\end{array}$ \\
\hline 1 & NCCP 13823(R) & 1.86 & $16(S)$ & $1,>16$ (IR) & $32(\mathrm{I})$ & $>16(\mathrm{R})$ & $16(\mathrm{I})$ & $8(\mathrm{I})$ & $8(\mathrm{R})$ & $>8,152(\mathrm{R})$ \\
\hline 3 & NCCP 13825(S) & 1.78 & $16(S)$ & $8(\mathrm{R})$ & $128(\mathrm{R})$ & $>16(\mathrm{R})$ & $>32(\mathrm{R})$ & $32(\mathrm{R})$ & $>8$ (R) & $>8,152$ (R) \\
\hline 4 & NCCP 13826(R) & 1.77 & $16(S)$ & $2,>16(\mathrm{R})$ & 64 (I) & $>16(\mathrm{R})$ & $32(\mathrm{R})$ & $16(\mathrm{I})$ & $>8(\mathrm{R})$ & $>8,152$ (R) \\
\hline 5 & NCCP 13827(S) & 2.44 & $8(\mathrm{~S})$ & $0.25,16$ (IR) & 64 (I) & $>16(\mathrm{R})$ & $4(\mathrm{~S})$ & $8(\mathrm{I})$ & $2(\mathrm{I})$ & $>8,152$ (R) \\
\hline 6 & NCCP 13828(S) & 2.63 & $8(S)$ & $1,>16$ (IR) & $32(\mathrm{I})$ & $>16(\mathrm{R})$ & $32(\mathrm{R})$ & $32(\mathrm{R})$ & $8(\mathrm{R})$ & $>8,152(\mathrm{R})$ \\
\hline 8 & NCCP 13839(S) & 1.86 & $8(S)$ & $2,>16(\mathrm{R})$ & $64(\mathrm{I})$ & $>16(\mathrm{R})$ & $32(\mathrm{R})$ & $16(\mathrm{I})$ & $>8(\mathrm{R})$ & $>8,152$ (R) \\
\hline 9 & NCCP 15798(R) & 2.24 & $8(S)$ & $0.25,16$ (IR) & 64 (I) & $>16$ (R) & $16(\mathrm{I})$ & $32(\mathrm{R})$ & $4(\mathrm{R})$ & $>8,152(\mathrm{R})$ \\
\hline
\end{tabular}

Abbreviation: susceptible (S), intermediate (I), inducible resistant (IR), resistant (R); antimicrobials and susceptibility breakpoints for non-tuberculous mycobacteria (NTM), as proposed by the Clinical \& Laboratory Standards Institute (CLSI 2011) [22] 
In addition, the anti-CLR variant was sensitive to $10-\mathrm{DEBC}$ as an $\mathrm{IC}_{50}$ band $(4.662 \sim 5.535 \mu \mathrm{g} / \mathrm{mL})$ and $\mathrm{MIC}_{90} 12.5 \mu \mathrm{g} / \mathrm{mL}$. It was the same regardless of whether it was a CLR-resistant or a susceptible strain. Thus, 10-DEBC should be an active drug to treat CLR-resistant $M$. abscessus. These results confirmed that 10-DEBC was active against clarithromycin-sensitive and -resistant strains. Therefore, 10-DEBC should be viewed as an active drug candidate for the treatment of clarithromycin-resistant $M$. abscessus.

\subsection{0-DEBC Is Susceptible to Nonreplicating and Biofilm Growing M. abscessus}

The activity of 10-DEBC in nonreplicating phase cultures was examined, and this phase, that was induced by lack of oxygen, was measured. Nonreplicating status was confirmed by measuring the growth curves of $M$. abscessus under aerobic and anaerobic conditions before assessing the drug action (data not shown) [27].

CLR showed increased activity against anaerobic cultured M. abscessus $\left(\mathrm{IC}_{50} 1.309 \mu \mathrm{g} / \mathrm{mL}\right.$ and $\mathrm{MIC}_{90}<10 \mu \mathrm{g} / \mathrm{mL}$ ) than aerobic condition $\left(\mathrm{IC}_{50} 0.034 \mu \mathrm{g} / \mathrm{mL}\right.$ and $\left.\mathrm{MIC}_{90} 0.078 \mu \mathrm{g} / \mathrm{mL}\right)$, demonstrating a significant 37.52-fold and 128.21-fold shift to higher $\mathrm{IC}_{50}$ values and $\mathrm{MIC}_{90}$ values (Figure 3). Interestingly, in an anaerobic state, the $\mathrm{IC}_{50}$ value of 10 -DEBC increased 1.18 times, and $\mathrm{MIC}_{90}$ of $10-\mathrm{DEBC}$ increased 2 times compared to the aerobic culture, showing similar results to the aerobic culture. Thus, 10-DEBC attained activity against anaerobic $M$. abscessus closely related to the nonreplicating environment.
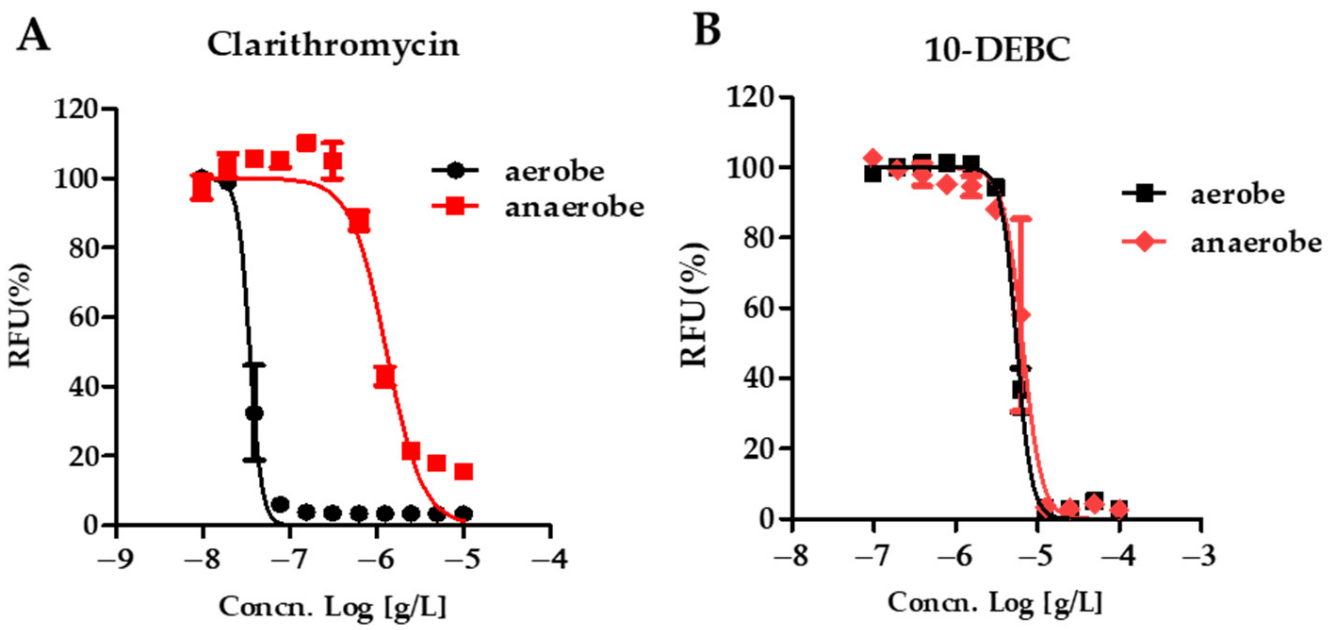

Figure 3. DRC of 10-DEBC. DRC of M. abscessus under aerobic, anaerobic using REMA. M. abscessus was undergone for seven days within an anaerobic generating container system (BD GasPak ${ }^{\mathrm{TM}} \mathrm{EZ}$ ). The activity of CLR (A) and 10-DEBC (B) against $M$. abscessus under aerobic and anaerobic conditions. The experiments were carried out with three biological replicates and expressed as the mean \pm SEM for each concentration.

Biofilm growth is a necessary factor in antibiotic resistance. Resistance to antibiotics, disinfectants, and biocides by biofilm-forming microorganisms can lead to treatment failure. Clinical experience has shown that biofilms must be removed to resolve the infection [28]. According to Muñoz-Egea, differences were found between MICs and minimum biofilm eradication concentration (MBEC) in M. abscessus; specifically, the MBEC of the CLR increased 100,000 times compared with the MIC [28]. However, when M. abscessus was exposed to CLR, MBEC was 6000-fold higher from 0.034 to $>200 \mu \mathrm{g}$ than the typical MIC of CLR.

As can be seen in Figure 4, CLR has excellent bactericidal activity $\left(\mathrm{IC}_{50}=0.034 \mu \mathrm{g} / \mathrm{mL}\right)$ in growing culture but lost its activity ultimately against biofilm-growing $M$. abscessus $\left(\mathrm{IC}_{50}>200 \mu \mathrm{g} / \mathrm{mL}\right)$. On the other side, 10 -DEBC still has an $\mathrm{IC}_{50}$ of $38.72 \mu \mathrm{g} / \mathrm{mL}$ and $\mathrm{MIC}_{90}$ of $50 \mu \mathrm{g} / \mathrm{mL}$ against biofilm-growing bacteria. It seems to be active against $M$. abscessus that grows on biofilms. These results suggest $10-\mathrm{DEBC}$ is an attractive substance for all types of anti-M. abscessus treatment. 

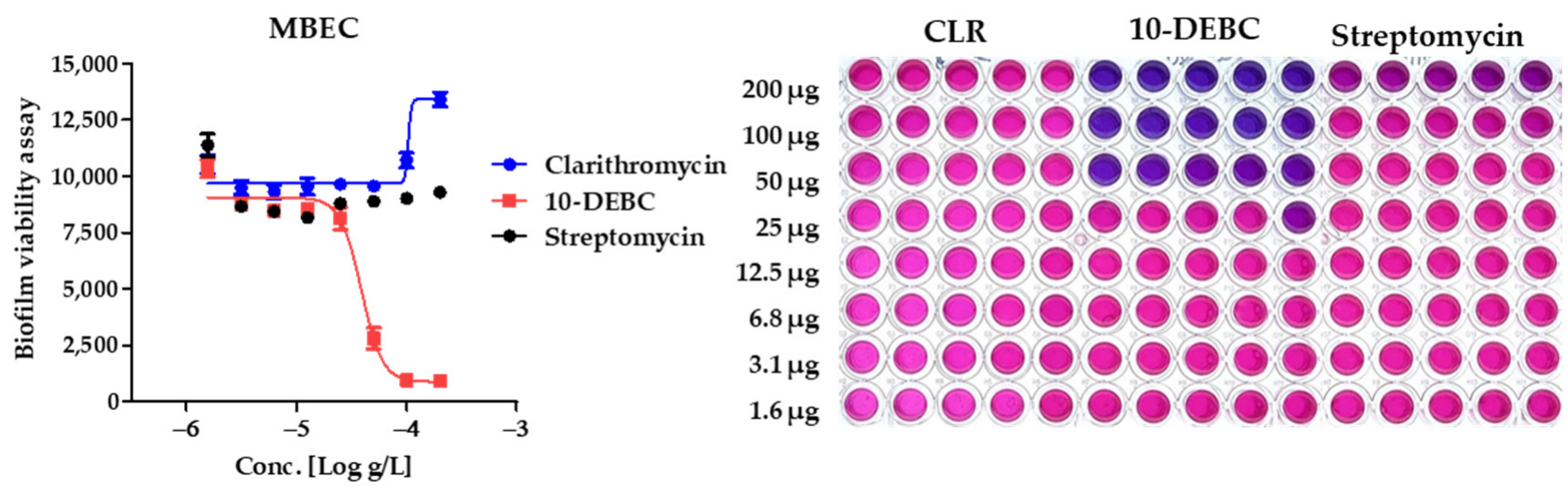

Figure 4. MBEC biofilm antimicrobial viability assay of 10-DEBC. The MBEC biofilm viability assay can confirm the effects of 10-DEBC-induced eradication on $M$. abscessus within biofilms by measuring the metabolic activities of live M. abscessus within biofilms using the resazurin. The experiments were carried out with three biological replicates and expressed as the mean \pm SEM for each concentration.

\subsection{0-DEBC Is Effective against Intracellular M. abscessus}

We assessed the cell viability at various concentrations to determine whether 10-DEBC affects cytotoxicity. 10-DEBC has not observed the cytotoxicity under particular concentrations that inhibited intracellular M. abscessus (data not shown) and did not show significant toxicity against macrophages differentiated from primary stem cells [21]. According to Janjetovic et al., as shown by LDH and MTT tests, 10-DEBC dihydrochloride at a concentration of $10 \mu \mathrm{M}$ did not affect cell viability when applied alone [19].

The dual reading test made it possible to obtain DRC for M. abscessus inhibition and cytotoxicity of THP1 cells [29]. $\mathrm{IC}_{50}$ and $\mathrm{CC}_{50}$ can be defined (cell toxicity concentration that induces $50 \%$ cell death) in this study. Figure 5 shows the dual DRC to 10-DEBC and the data obtained from the same screening wells over time. Cytotoxicity was measured with the SYTO 60 probe. Treatment with CLR successfully rescued cells from infection challenge and prevented bacterial replication (data not shown). Kim et al. showed that CLR significantly reduced the intracellular $M$. abscessus present two days after infection at concentrations of $0.1 \mu \mathrm{g} / \mathrm{mL}$ [30]. 10-DEBC inhibited the growth of M. abscessus (lux) in a dual read assay with an $\mathrm{IC}_{50}$ of $3.48 \mu \mathrm{g} / \mathrm{mL}$, with no cytotoxicity for THP1, even at the highest concentrations (Figure 5A). Consistently with the THP-1 infection model, the intracellular inhibitory activity of 10-DEBC was shown (Figure 5B). 10-DEBC was found to inhibit the intracellular growth of M. abscessus in THP-1 cells with an $\mathrm{IC}_{50}$ of $13.18 \mu \mathrm{g} / \mathrm{mL}$ (Figure 5B), while inhibiting replication of $M$. abscessus inside human embryonic cellderived macrophages (iMACs) with an $\mathrm{IC}_{50}$ of $5.8 \mu \mathrm{g} / \mathrm{mL}$, without host cell toxicity even at the highest concentration of $50 \mu \mathrm{g} / \mathrm{mL}$ (Figure $5 \mathrm{C}, \mathrm{D}$ ).

\subsection{0-DEBC Is Penetrated in Caseum Surrogate}

The caseum surrogate binding assay is a valuable tool in drug discovery for tuberculosis and NTM and is used in many lead optimization programs. The higher the $f u$, the more the drug diffuses into caseum. To avoid collecting caseum from infected animals, we designed a caseum simulation that will mimic the binding properties of a real matrix.

THP-1 monocytes were exposed to $94 \mu \mathrm{g} / \mathrm{mL}$ oleic acid and $94 \mu \mathrm{g} / \mathrm{mL}$ linoleic acid, which induce differentiation into foamy macrophage (FM) in vitro [31]. PMA-differentiated THP-1 macrophages (THPM) accumulated lipid droplets most significantly in the presence of $94 \mu \mathrm{g} / \mathrm{mL}$ oleic acid and $94 \mu \mathrm{g} / \mathrm{mL}$ linoleic acid. THPMs were treated with oleic acid and linoleic acid for $24 \mathrm{~h}$, and the resulting FM were infected, washed, harvested, lysed, and denatured before being used in the RED assay. 
A

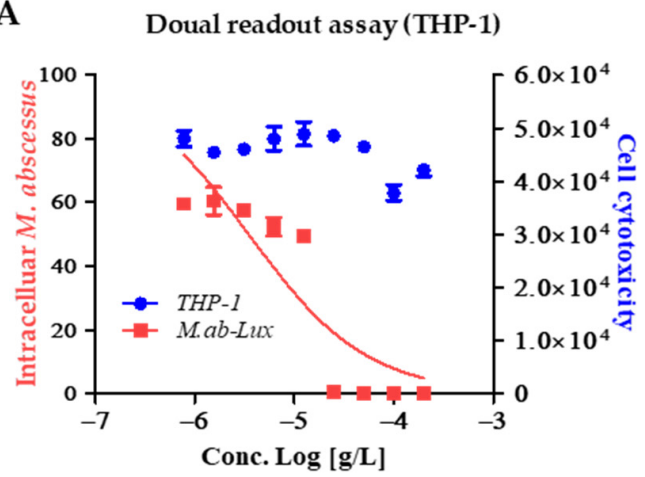

D

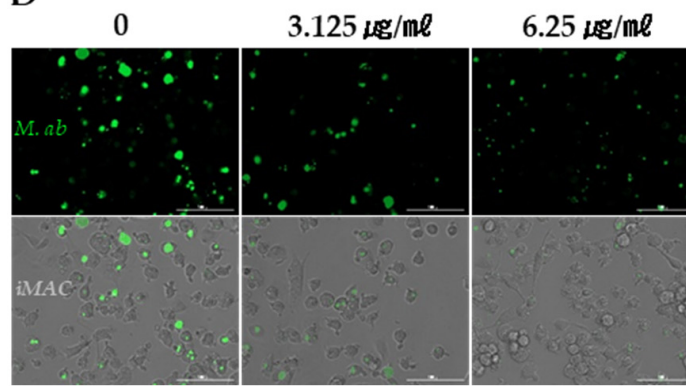

B

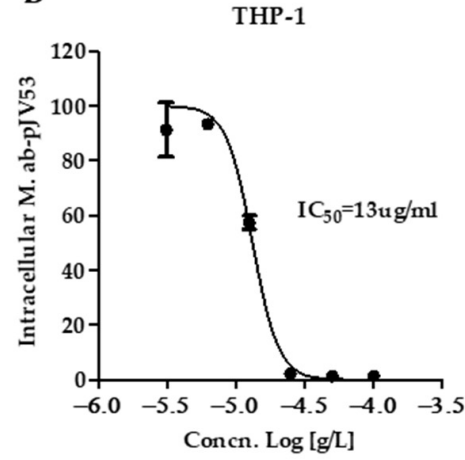

$12.5 \mu \mathrm{g} / \mathrm{m} \ell$
C

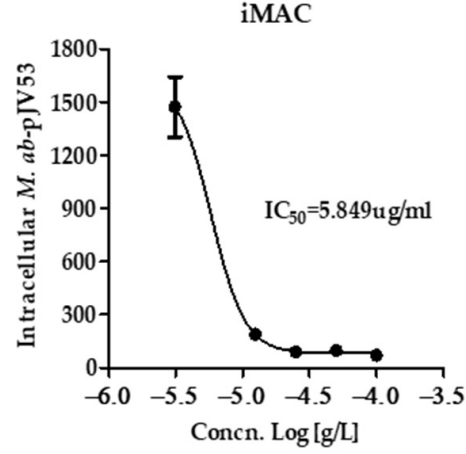

$50 \nu \mathrm{g} / \mathrm{m} \ell$

$100 \mathrm{ug} / \mathrm{m} \ell$
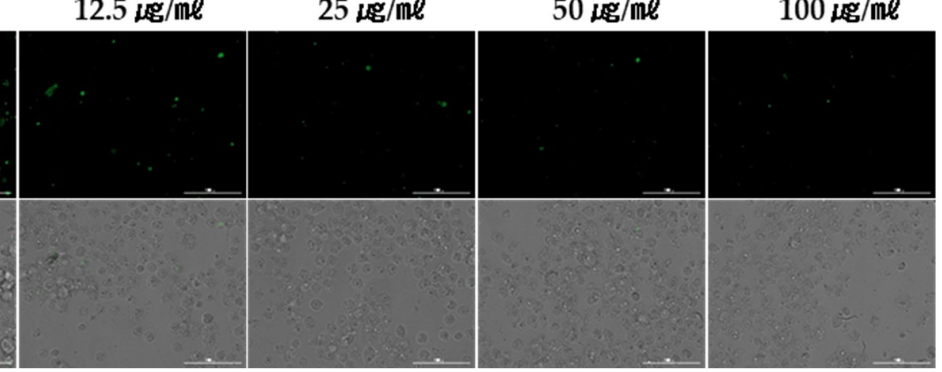

Figure 5. 10-DEBC promotes the killing of intracellular M. abscessus. (A) Dual reading assay of 10-DEBC within macrophages. Infections in macrophages were carried out with multiplicities of infection (MOI) of 2 bacteria per cell for three hours and washed to remove extracellular mycobacteria. Luminescence detected from luciferase-expressing M. abscessus alone within THP-1 macrophages. The infected THP-1 cells stained with SYTO 60 were counted under fluorescence detection, and total cells were counted using Multi Reader. (B) THP-1 activated with PMA were infected at MOI of 2 with GFP-expressed M. abscessus for three hours, followed by treatment with 10-DEBC indicated concentrations in fresh medium. Ratio sum intensity values were used for all data reduction steps. (C) iMACs were infected at MOI of 2 with GFP-expressed M. abscessus for three hours, followed by treatment with 10-DEBC indicated concentrations in fresh medium. (D) Images of GFP-expressing M. abscessus infected iMACs on day three after treatment with indicated concentrations of 10-DEBC. Automated microscopy using a Lionheart ${ }^{\mathrm{TM}}$ automated live-cell imager. The Gen $5^{\mathrm{TM}} 3.05$ software object feature enables the identification of cells within the imaging field. Data were expressed as the mean \pm SD of triplication for each concentration. (Scale bar $=100 \mu \mathrm{m}$.).

The drug concentration in the donor and receiver chamber of the RED assay was quantified by LC-MS. KBIO (Osong, Korea) conducted this experiment. Drug quantification was achieved using liquid chromatography coupled to mass spectrometry (LC-MS) methods (Figure S1). The LC-MRM strategy was used for quantification of drugs in the donor and receiver chamber of RED. Respective parameters determined for analytes used herein are shown in Supplementary Table S1.

The fraction unbound of CLR and 10-DEBC were determined in matrixes with triplicate samples for each batch (Table 2). The mean fu (\%) for CLR was 39.92\% in the surrogate matrix, and undiluted $f u$ was 86.91 . The 10-DEBC's average caseum-free fraction (caseum $f u \%$ ) of $0.04 \%$ to $0.06 \%$ (standard deviation (SD), $0.005 \%$ ) and undiluted $f u$ of 5.696 was much lower than CLR's caseum fraction.

Table 2. Fraction unbound $\left(f_{u}\right)$ of CLR and 10-DEBC in caseum surrogate binding assay.

\begin{tabular}{cccc}
\hline & $f u$ & $f u(\%)$ & Undiluted $f u$ \\
\hline CLR & $0.40 \pm 0.01$ & $39.92 \pm 1.22$ & $86.91 \pm 0.58$ \\
10-DEBC & $0.0060 \pm 0.0005$ & $0.60 \pm 0.054$ & $5.696 \pm 0.501$ \\
\hline
\end{tabular}

All results are expressed as the mean \pm standard deviation. 


\section{Discussion}

Pulmonary NTM is a recognized disease in developed countries such as Japan [32] and USA [33]. Opposite trends in NTM and tuberculosis rates were observed in 75\% of 16 regions on four continents [34]. When talking about diseases caused by NTM, it remains tough and especially involves $M$. abscessus $[35,36]$. It is challenging to treat $M$. abscessus like other NTMs; it is resistant to most antibiotics, including macrolides, aminoglycosides, rifamycins, tetracyclines, and $\beta$-lactams. There is no consistent regimen with demonstrated or predicted efficacy for pulmonary disease caused by M. abscessus. Unfortunately, despite combined treatment, success rates only range from $25 \%$ to $42 \%$ [37]. Approximately $20 \%$ of M. abscessus strains isolated from pulmonary infections do not respond to the macrolides included in treatment $[38,39]$. Therefore, there is an urgent medical need to discover and develop new and more effective anti-M. abscessus drugs.

It is already known that the $\mathrm{R}$ phenotype is much more virulent than $\mathrm{S}$ morphology and tends to cause very persistent and difficult-to-treat infections $[25,40]$. The $\mathrm{R}$ form is involved in chronic airway colonization in CF [24]. 10-DEBC is effective in vitro against both the reference strain and the clinically isolated strains of $\mathrm{R}$ and $\mathrm{S}$ colony morphologies in our study. In this regard, 10-DEBC has additional clinical benefits.

CLR is an essential antibiotic for treating M. abscessus infections, but intrinsic and acquired resistance to CLR make treatment increasingly tricky and have unsatisfactory outcomes [41]. This is common in clinical isolates of M. abscessus, especially clinical Rtype strains. We used spontaneous CLR-resistant mutants to test whether 10-DEBC is effective against CLR-resistant strains. Additionally, these CLR-resistant mutants show high drug resistance to CLR. Our in vitro experimental results, as shown in Figure 2 and Table 1, suggest that 10-DEBC is functioning to susceptible, induced CLR-resistant, intrinsic CLR-resistant, and clinical R morphotype M. abscessus strains. Our experimental results demonstrate that 10 -DEBC has a suggestive advantage in being used as a core drug candidate for $M$. abscessus disease.

In a recent transcriptomic study, the total $M$. abscessus DosR regulon increased under hypoxia [42]. DosR, a modulator of the resting survival response necessary for the survival of tubercle bacilli under hypoxia, is maintained in all NTM species [43]. The ability of M. abscessus to generate biofilm represents a successful survival strategy for these ubiquitous microorganisms. They form a biofilm on the airways' surface inside the human lung [44].

It suggests that $M$. abscessus have the conserved molecular strategies for persisting inside the host. As the disease evolves, M. abscessus may not replicate and exhibit phenotypic drug resistance within lung lesions, which may be one of the factors contributing to the persistence of $M$. abscessus infection during long-term treatment $[45,46]$. This behavior is related to their pathogenicity and increased antibiotic resistance $[47,48]$. In general, the drug effort is established against $M$. abscessus active during aerobic growth. Consequently, treatment outcomes vary with the use of the same antibiotics against different bacilli phenotypes, and they are often ineffective. Most M. abscessus remain nonrenewable and are in a low metabolic state in granulomas and biofilm formation in the pulmonary airways. Situations in the host are entirely different from in vitro testing for conventional antibiotic activities. In this study, we examined the efficacy of 10-DEBC against different phenotypes of M. abscessus, which survived in biofilm and anaerobic environments as found in the lungs of patients. The superiority of 10-DEBC is that it is effective even under adverse anoxic and biofilm conditions.

NTMs can grow and survive outside and within cells, such as inside macrophages. In pulmonary infection with NTM, the bacilli attract the mucosa and are phagocytosed by macrophages. Infected macrophage cell lines may exhibit physiological conditions mimicking actual NTM disease with resistance to M. abscessus. As shown in Figure 5, 10-DEBC inhibited the growth of $M$. abscessus in macrophage and macrophage cell lines derived from human embryonic cells. 
The discovery of more effective anti-TB drugs requires a deeper understanding of the different mechanisms of drug penetration into the envelope of tuberculous lesions because granulomas and necrotic niches both contain caseum, which is difficult to sterilize [49]. Rapid equilibrium dialysis has been used to measure drug binding in a small amount of caseum as it has been validated with various tissue homogenates [50]. Mass balance was systematically included to exclude the nonspecific binding of drugs to the device.

A caseum assay might be challenging to develop for NTM drug screening because caseum-producing animal models are not well established yet. Alternately, we used the caseum surrogate assay designed from artificial, cell-culture-based caseum. We tested in the surrogate caseum. The very strongly bound $(f u \leq 1 \%)$ compounds were almost entirely retained in the chamber for no effective penetration into the caseum. Meanwhile, less bound compounds $(1 \%<f u<100 \%)$ can diffuse into the nucleus to varying degrees; the higher the $f u$, the more it penetrates the necrotic area.

As shown in Table 2, the unbound fraction of 10-DEBC is lower than CLR. Sarathy et al. [51] showed that bedaquiline and clofazimine are highly bound compounds $(f u \leq 0.01 \%$ ). Bedaquiline and clofazimine are almost entirely trapped within the donor chamber and are not effective at permeating the caseum.

10-DEBC was first reported as a cell-permeable phenoxazine-derivative inhibitor of AKT kinase phosphorylation [52]. Activating the AKT1 signaling promotes intracellular Mycobacterium tuberculosis (Mtb) survival in macrophages through several mechanisms, including inhibition of phagosome-lysosome fusion [53]. It probably applies to M. abscessus as expected. 10-DEBC is a member of the chlorpromazine family of compounds, which were demonstrated to inhibit Mtb [54] without being Akt inhibitors themselves. 10-DEBC appears to be enriched by the macrophages and has been suggested to bind calmodulin and interfere with calcium transport [55]. Therefore, 10-DEBC may inhibit M. abscessus growth by interfering with not yet revealed mechanisms. Further research is needed to characterize the mechanisms by which 10-DEBC mediates intracellular and extracellular death of M. abscessus.

In conclusion, the results of this study on 10-DEBC hydrochloride activity suggest that this is a potent new drug candidate against $M$. abscessus infection.

\section{Materials and Methods}

\subsection{Bacterial Strains and Culture Conditions}

The M. abscessus strain (DSMZ 44196) was grown at $37^{\circ} \mathrm{C}$ in Middlebrook $7 \mathrm{H} 9$ broth (BD, 27130) supplemented with 10\% OADC (BD, 212240). We obtained the nine clinical strains from the National Culture Collection for Pathogens (NCCP). These strains were sent to the Seoul Clinical Laboratory (SCL) for conventional microbiological and biochemical tests. SCL provided the mycobacterial identification results. Except for 10-DEBC, MICs for nine drugs were formed with the broth microdilution method (SCL's guideline, Yongin-si, Gyeonggi-do, Korea).

M. abscessus CLR-resistant mutant was prepared as previously described [27]. Recombinant $M$. abscessus expressing green fluorescence protein and luminescence protein for the macrophage infection were prepared as previously described [27]. The nonreplicating condition was previously described [27]. 10-[4'-(N, N-Diethylamino)butyl]-2-chlorophenoxazine (10-DEBC) was obtained from Tocris Bioscience (Bristol, UK).

\subsection{DRC Testing}

The MICs were determined according to the CLSI guidelines. We treated 10-DEBC in actively growing $M$. abscessus, $M$. abscessus-pLUX G13, CLR-R mutant, and anaerobic cultured $M$. abscessus.

10-DEBC was diluted two-fold in a serial dilution in 96-well plates with a total volume of $100 \mathrm{uL}$. It was then incubated for $84 \mathrm{~h}$ at $37^{\circ} \mathrm{C}$ before adding $0.025 \%$ resazurin reagent. After overnight incubation, the fluorescence of resorufin (metabolite resazurin) was determined using Synergy H1 Hybrid Multi-Mode Reader (Bio-Tek, Winooski, VT, USA). IC 50 
values were calculated from the raw fluorescence data using Prism 5.0 software (GraphPad Inc., La Jolla, CA, USA). The experiments were carried out with triplicates.

\subsection{Biofilm Assays}

The MBEC assay was previously described [27]. The biofilm formation was conducted and the 96-peg lid was placed onto a 96-well. Next, we prepared $200 \mu \mathrm{L}$ 10-DEBC solutions with the medium and then added them to each well of the 96-well plate, leaving them for four days. Following incubation, MBEC was quantified by performing the optimized resazurin reagents.

\subsection{Intracellular Killing Assay}

THP-1 cells were treated with a final concentration of $50 \mathrm{nM}$ PMA (Sigma Aldrich, St. Louis, MO, USA) for two days. Infection with M. abscessus harboring green fluorescent (pTEC15) or luciferase was carried out at $37^{\circ} \mathrm{C}$ for three hours at an MOI of 2:1. After three washes with PBS, cells were incubated containing $50 \mu \mathrm{g} / \mathrm{L}$ gentamycin for $30 \mathrm{~min}$ and washed again. Then, $200 \mathrm{uL}$ RPMI 1640 media containing the indicated concentration of 10-DEBC was left for four days. The macrophages were stained with SYTO 60 (Invitrogen, Waltham, MA, USA) dye at a final concentration of $5 \mu \mathrm{M}$ for $30 \mathrm{~min}$ at $37^{\circ} \mathrm{C}$. Luminescence was measured on day four using a Synergy H1 microplate reader (Bio-Tek, Winooski, VT, USA).

iMAC cells were previously described and provided kindly by Dr. Jung-Hyun Kim [21]. The iMAC cell's infection method using M. abscessus harboring green fluorescent was the same as differentiated THP- 1 cells. Live-cell images were captured by automated microscopy using Lionheart ${ }^{\mathrm{TM}} \mathrm{FX}$ automated microscopy (Bio-Tek, Winooski, VT, USA). The Agilent BioTek Gen $5^{\mathrm{TM}} 3.05$ software object feature enables the calculation of cells within the imaging field.

\subsection{Generation of Surrogate Caseum}

The THP-1 cells were seeded on a $150 \mathrm{~mm}$ dish, exposed to $100 \mathrm{nM}$ PMA, and allowed to adhere. We used lipid body production in THP-1 monocytes to simulate caseum formation. This experiment was carried out overnight with $100 \mathrm{X}$ Linoleic acid-Oleic acid-Albumin (LOA) (Sigma L9655), which is known to induce differentiation into foamy macrophage in vitro [31]. Infection with $M$. abscessus was carried out at an MOI of 5:1. After three washes with PBS, cells were treated $50 \mu \mathrm{g} / \mathrm{L}$ gentamycin for $30 \mathrm{~min}$ and washed again. They were then incubated with RPMI 1640 media, having LOA for one day. Infected foamy macrophages were lysed during three freezing-thaw cycles. A 30 min incubation denatured proteins at $75^{\circ} \mathrm{C}$ to mimic alterations in protein structure and $3 \mathrm{D}$ conformation in the necrotic core of granulomas.

All binding assays were conducted using the RED device (Thermo Fisher Scientific, Waltham, MA, USA) and were previously described [56]. Using liquid chromatography coupled to mass spectrometry (LC-MS) methods, drug quantification was achieved. Mass analysis and detection were performed on a SCIEX (Framingham, MA, USA) 6500 Q-trap triple-quadrupole mass spectrometer equipped with a turbo ion-spray ionization source. The HPLC system was Agilent (Santa Clara, CA, USA) 1290 series with a degasser, binary pump, autosampler, and thermostat column compartment. Chromatographic separation for all compounds was achieved with an Agilent Poroshell $(2.1 \times 50 \mathrm{~mm}, 2.7 \mu \mathrm{m})$ column using gradient conditions. Exponential gradient elution $(10 \mathrm{~min})$ increased the mobile phase composition from 0 to $95 \%$ Sol B. The gradient was then decreased to $5 \%$ B to equilibrate the column for the next run. The compounds were directly injected with $2 \mu \mathrm{L}$ of our prepared sample with Sol A ( $1 \mathrm{mM}$ ammonium acetate in DW and $0.1 \%$ FA) and Sol B ( $1 \mathrm{mM}$ ammonium acetate in methanol and $0.1 \% \mathrm{FA}$ ) into the analytical column without a trap column; the flow rate was set to $0.4 \mathrm{uL} / \mathrm{min}$. Data collection and processing were performed with Mass Hunter Quantitative Analysis software. The unbound fractions were calculated using previous studies' methods [57,58]. 
The $f \mathrm{u}$ in diluted surrogate caseum was calculated as the ratio between buffer chamber and sample chamber concentrations, as shown in Equation (1). A dilution factor of 10 $(\mathrm{D}=10)$ was applied to calculate $f \mathrm{u}$ in undiluted surrogate caseum, as shown in Equation (2) [58].

$$
\begin{gathered}
f u=\frac{[\text { Receiver Concentation }]}{[\text { Donor Concentration }]} \\
\text { undiluted } f u=\frac{1 / D}{\left(\left(\frac{1}{f u}\right)-1\right)+1 / D}
\end{gathered}
$$

\subsection{Ethics}

The Institutional Review Board approved all studies of Masan National Tuberculosis Hospital (IRB-398837-2018-E34, approved on 14 January 2019) and the Institutional Biosafety Committees (MTHIBC-19-01 and MTHIBC-21-11, approved on 26 February 2019 and 15 July 2021). The ethical use of hESC was approved by IRB of Korea Centers for Disease Control and Prevention: approval number 2020-02-09-C-A.

Supplementary Materials: The following supporting information can be downloaded at: https: / / www.mdpi.com/article/10.3390/ijms23020591/s1.

Author Contributions: D.-G.L. and S.R. designed the study and experiments. Y.H., Y.L., H.-J.K., J.H. and J.-H.K. performed the experiments and generated the data. D.-G.L. and S.R. analyzed the data and wrote the manuscript. All authors have read and agreed to the published version of the manuscript.

Funding: This research was supported by a grant of the Korea Health Technology R\&D Project through the Korea Health Industry Development Institute (KHIDI), funded by the Ministry of Health and Welfare, Republic of Korea (grant number: HI20C0478).

Institutional Review Board Statement: The study was conducted according to the guidelines of the Declaration of Helsinki and approved by the Institutional Review Board of Masan National Tuberculosis Hospital (IRB-398837-2018-E34, 14 January 2019). The ethical use of hESC was approved by IRB of Korea Centers for Disease Control and Prevention: approval number 2020-02-09-C-A.

Informed Consent Statement: Not applicable.

Data Availability Statement: Not applicable.

Conflicts of Interest: The authors have no conflict of interest to declare.

\section{References}

1. Falkinham, J.O., 3rd. Environmental sources of nontuberculous mycobacteria. Clin. Chest Med. 2015, 36, 35-41. [CrossRef]

2. Brown-Elliott, B.A.; Wallace, R.J., Jr. Clinical and taxonomic status of pathogenic nonpigmented or late-pigmenting rapidly growing mycobacteria. Clin. Microbiol. Rev. 2002, 15, 716-746. [CrossRef]

3. Alcaide, F.; Pena, M.J.; Perez-Risco, D.; Camprubi, D.; Gonzalez-Luquero, L.; Grijota-Camino, M.D.; Dorca, J.; Santin, M. Increasing isolation of rapidly growing mycobacteria in a low-incidence setting of environmental mycobacteria, 1994-2015. Eur. J. Clin. Microbiol. Infect. Dis. 2017, 36, 1425-1432. [CrossRef] [PubMed]

4. Griffith, D.E.; Aksamit, T.; Brown-Elliott, B.A.; Catanzaro, A.; Daley, C.; Gordin, F.; Holland, S.M.; Horsburgh, R.; Huitt, G.; Iademarco, M.F.; et al. An official ATS/IDSA statement: Diagnosis, treatment, and prevention of nontuberculous mycobacterial diseases. Am. J. Respir. Crit. Care Med. 2007, 175, 367-416. [CrossRef] [PubMed]

5. Tortoli, E. Clinical manifestations of nontuberculous mycobacteria infections. Clin. Microbiol. Infect. 2009, 15, 906-910. [CrossRef] [PubMed]

6. Sfeir, M.; Walsh, M.; Rosa, R.; Aragon, L.; Liu, S.Y.; Cleary, T.; Worley, M.; Frederick, C.; Abbo, L.M. Mycobacterium abscessus Complex Infections: A Retrospective Cohort Study. Open Forum Infect. Dis. 2018, 5, ofy022. [CrossRef] [PubMed]

7. Hayes, D., Jr. Mycobacterium abscessus and other nontuberculous mycobacteria: Evolving respiratory pathogens in cystic fibrosis: A case report and review. South. Med. J. 2005, 98, 657-661. [CrossRef]

8. Olivier, K.N.; Weber, D.J.; Wallace, R.J., Jr.; Faiz, A.R.; Lee, J.H.; Zhang, Y.; Brown-Elliot, B.A.; Handler, A.; Wilson, R.W.; Schechter, M.S.; et al. Nontuberculous mycobacteria. I: Multicenter prevalence study in cystic fibrosis. Am. J. Respir. Crit. Care Med. 2003, 167,828-834. [CrossRef] 
9. Sermet-Gaudelus, I.; Le Bourgeois, M.; Pierre-Audigier, C.; Offredo, C.; Guillemot, D.; Halley, S.; Akoua-Koffi, C.; Vincent, V.; Sivadon-Tardy, V.; Ferroni, A.; et al. Mycobacterium abscessus and children with cystic fibrosis. Emerg. Infect. Dis. 2003, 9, 1587-1591. [CrossRef]

10. Qvist, T.; Taylor-Robinson, D.; Waldmann, E.; Olesen, H.V.; Hansen, C.R.; Mathiesen, I.H.; Hoiby, N.; Katzenstein, T.L.; Smyth, R.L.; Diggle, P.J.; et al. Comparing the harmful effects of nontuberculous mycobacteria and Gram negative bacteria on lung function in patients with cystic fibrosis. J. Cyst. Fibros. 2016, 15, 380-385. [CrossRef]

11. Jonsson, B.E.; Gilljam, M.; Lindblad, A.; Ridell, M.; Wold, A.E.; Welinder-Olsson, C. Molecular epidemiology of Mycobacterium abscessus, with focus on cystic fibrosis. J. Clin. Microbiol. 2007, 45, 1497-1504. [CrossRef] [PubMed]

12. Esther, C.R., Jr.; Esserman, D.A.; Gilligan, P.; Kerr, A.; Noone, P.G. Chronic Mycobacterium abscessus infection and lung function decline in cystic fibrosis. J. Cyst. Fibros. 2010, 9, 117-123. [CrossRef]

13. Catherinot, E.; Roux, A.L.; Macheras, E.; Hubert, D.; Matmar, M.; Dannhoffer, L.; Chinet, T.; Morand, P.; Poyart, C.; Heym, B.; et al. Acute respiratory failure involving an R variant of Mycobacterium abscessus. J. Clin. Microbiol. 2009, 47, 271-274. [CrossRef] [PubMed]

14. Taylor, J.L.; Palmer, S.M. Mycobacterium abscessus chest wall and pulmonary infection in a cystic fibrosis lung transplant recipient. J. Heart Lung Transpl. 2006, 25, 985-988. [CrossRef] [PubMed]

15. Kwak, N.; Dalcolmo, M.P.; Daley, C.L.; Eather, G.; Gayoso, R.; Hasegawa, N.; Jhun, B.W.; Koh, W.J.; Namkoong, H.; Park, J.; et al. M ycobacterium abscessus pulmonary disease: Individual patient data meta-analysis. Eur. Respir. J. 2019, 54, 1801991. [CrossRef]

16. Kim, S.Y.; Shin, S.J.; Jeong, B.H.; Koh, W.J. Successful antibiotic treatment of pulmonary disease caused by Mycobacterium abscessus subsp. abscessus with C-to-T mutation at position 19 in erm(41) gene: Case report. BMC Infect. Dis. 2016, 16, 207. [CrossRef] [PubMed]

17. Tsai, S.H.; Shen, G.H.; Lin, C.H.; Liau, J.R.; Lai, H.C.; Hu, S.T. Mab_3168c, a putative acetyltransferase, enhances adherence, intracellular survival and antimicrobial resistance of Mycobacterium abscessus. PLoS ONE 2013, 8, e67563. [CrossRef]

18. Stout, J.E.; Koh, W.J.; Yew, W.W. Update on pulmonary disease due to non-tuberculous mycobacteria. Int. J. Infect. Dis. 2016, 45, 123-134. [CrossRef]

19. Janjetovic, K.; Vucicevic, L.; Misirkic, M.; Vilimanovich, U.; Tovilovic, G.; Zogovic, N.; Nikolic, Z.; Jovanovic, S.; Bumbasirevic, V.; Trajkovic, V.; et al. Metformin reduces cisplatin-mediated apoptotic death of cancer cells through AMPK-independent activation of Akt. Eur. J. Pharm. 2011, 651, 41-50. [CrossRef]

20. Brunet, A.; Bonni, A.; Zigmond, M.J.; Lin, M.Z.; Juo, P.; Hu, L.S.; Anderson, M.J.; Arden, K.C.; Blenis, J.; Greenberg, M.E. Akt promotes cell survival by phosphorylating and inhibiting a Forkhead transcription factor. Cell 1999, 96, 857-868. [CrossRef]

21. Han, H.W.; Seo, H.H.; Jo, H.Y.; Han, H.J.; Falcao, V.C.A.; Delorme, V.; Heo, J.; Shum, D.; Choi, J.H.; Lee, J.M.; et al. Drug Discovery Platform Targeting M. tuberculosis with Human Embryonic Stem Cell-Derived Macrophages. Stem Cell Rep. 2019, 13, 980-991. [CrossRef]

22. Woods, G.L.; Brown-Elliott, B.A.; Conville, P.S.; Desmond, E.P.; Hall, G.S.; Lin, G.; Pfyffer, G.E.; Ridderhof, J.C.; Siddiqi, S.H.; Wallace, R.J., Jr.; et al. CLSI Standards: Guidelines for Health Care Excellence. In Susceptibility Testing of Mycobacteria, Nocardiae, and Other Aerobic Actinomycetes; Clinical and Laboratory Standards Institute: Wayne, PA, USA, 2011.

23. Andreu, N.; Fletcher, T.; Krishnan, N.; Wiles, S.; Robertson, B.D. Rapid measurement of antituberculosis drug activity in vitro and in macrophages using bioluminescence. J. Antimicrob. Chemother. 2012, 67, 404-414. [CrossRef] [PubMed]

24. Sanguinetti, M.; Ardito, F.; Fiscarelli, E.; La Sorda, M.; D’Argenio, P.; Ricciotti, G.; Fadda, G. Fatal pulmonary infection due to multidrug-resistant Mycobacterium abscessus in a patient with cystic fibrosis. J. Clin. Microbiol. 2001, 39, 816-819. [CrossRef]

25. Byrd, T.F.; Lyons, C.R. Preliminary characterization of a Mycobacterium abscessus mutant in human and murine models of infection. Infect. Immun. 1999, 67, 4700-4707. [CrossRef] [PubMed]

26. Nash, K.A.; Brown-Elliott, B.A.; Wallace, R.J., Jr. A novel gene, erm(41), confers inducible macrolide resistance to clinical isolates of Mycobacterium abscessus but is absent from Mycobacterium chelonae. Antimicrob. Agents Chemother. 2009, 53, 1367-1376. [CrossRef]

27. Lee, D.-G.; Hwang, Y.-H.; Park, E.-J.; Kim, J.-H.; Ryoo, S.-W. Clomiphene Citrate Shows Effective and Sustained Antimicrobial Activity against Mycobacterium abscessus. Int. J. Mol. Sci. 2021, 22, 11029. [CrossRef] [PubMed]

28. Munoz-Egea, M.C.; Garcia-Pedrazuela, M.; Mahillo, I.; Esteban, J. Effect of ciprofloxacin in the ultrastructure and development of biofilms formed by rapidly growing mycobacteria. BMC Microbiol. 2015, 15, 18. [CrossRef]

29. Stanley, S.A.; Barczak, A.K.; Silvis, M.R.; Luo, S.S.; Sogi, K.; Vokes, M.; Bray, M.A.; Carpenter, A.E.; Moore, C.B.; Siddiqi, N.; et al. Identification of host-targeted small molecules that restrict intracellular Mycobacterium tuberculosis growth. PLoS Pathog. 2014, 10, e1003946. [CrossRef]

30. Kim, T.S.; Choe, J.H.; Kim, Y.J.; Yang, C.S.; Kwon, H.J.; Jeong, J.; Kim, G.; Park, D.E.; Jo, E.K.; Cho, Y.L.; et al. Activity of LCB01-0371, a Novel Oxazolidinone, against Mycobacterium abscessus. Antimicrob. Agents Chemother. 2017, 61, e02752-16. [CrossRef]

31. den Hartigh, L.J.; Connolly-Rohrbach, J.E.; Fore, S.; Huser, T.R.; Rutledge, J.C. Fatty acids from very low-density lipoprotein lipolysis products induce lipid droplet accumulation in human monocytes. J. Immunol. 2010, 184, 3927-3936. [CrossRef]

32. Namkoong, H.; Kurashima, A.; Morimoto, K.; Hoshino, Y.; Hasegawa, N.; Ato, M.; Mitarai, S. Epidemiology of Pulmonary Nontuberculous Mycobacterial Disease, Japan. Emerg. Infect. Dis. 2016, 22, 1116-1117. [CrossRef] [PubMed] 
33. Lin, C.; Russell, C.; Soll, B.; Chow, D.; Bamrah, S.; Brostrom, R.; Kim, W.; Scott, J.; Bankowski, M.J. Increasing Prevalence of Nontuberculous Mycobacteria in Respiratory Specimens from US-Affiliated Pacific Island Jurisdictions(1). Emerg. Infect. Dis. 2018, 24, 485-491. [CrossRef] [PubMed]

34. Brode, S.K.; Daley, C.L.; Marras, T.K. The epidemiologic relationship between tuberculosis and non-tuberculous mycobacterial disease: A systematic review. Int. J. Tuberc. Lung Dis. 2014, 18, 1370-1377. [CrossRef]

35. Chen, J.; Zhao, L.; Mao, Y.; Ye, M.; Guo, Q.; Zhang, Y.; Xu, L.; Zhang, Z.; Li, B.; Chu, H. Clinical Efficacy and Adverse Effects of Antibiotics Used to Treat Mycobacterium abscessus Pulmonary Disease. Front. Microbiol. 2019, 10, 1977. [CrossRef]

36. Davidson, R.M. A Closer Look at the Genomic Variation of Geographically Diverse Mycobacterium abscessus Clones That Cause Human Infection and Disease. Front. Microbiol. 2018, 9, 2988. [CrossRef]

37. Nessar, R.; Cambau, E.; Reyrat, J.M.; Murray, A.; Gicquel, B. Mycobacterium abscessus: A new antibiotic nightmare. J. Antimicrob. Chemother. 2012, 67, 810-818. [CrossRef]

38. Bastian, S.; Veziris, N.; Roux, A.L.; Brossier, F.; Gaillard, J.L.; Jarlier, V.; Cambau, E. Assessment of clarithromycin susceptibility in strains belonging to the Mycobacterium abscessus group by erm(41) and rrl sequencing. Antimicrob. Agents Chemother. 2011, 55, 775-781. [CrossRef]

39. Hurst-Hess, K.; Rudra, P.; Ghosh, P. Mycobacterium abscessus WhiB7 Regulates a Species-Specific Repertoire of Genes To Confer Extreme Antibiotic Resistance. Antimicrob. Agents Chemother. 2017, 61, e01347-17. [CrossRef] [PubMed]

40. Howard, S.T.; Rhoades, E.; Recht, J.; Pang, X.; Alsup, A.; Kolter, R.; Lyons, C.R.; Byrd, T.F. Spontaneous reversion of Mycobacterium abscessus from a smooth to a rough morphotype is associated with reduced expression of glycopeptidolipid and reacquisition of an invasive phenotype. Microbiology 2006, 152, 1581-1590. [CrossRef]

41. Sharma, M.K.; La, Y.; Janella, D.; Soualhine, H. A real-time PCR assay for rapid identification of inducible and acquired clarithromycin resistance in Mycobacterium. abscessus. BMC Infect. Dis. 2020, 20, 944. [CrossRef]

42. Miranda-CasoLuengo, A.A.; Staunton, P.M.; Dinan, A.M.; Lohan, A.J.; Loftus, B.J. Functional characterization of the Mycobacterium abscessus genome coupled with condition specific transcriptomics reveals conserved molecular strategies for host adaptation and persistence. BMC Genom. 2016, 17, 553. [CrossRef] [PubMed]

43. Gerasimova, A.; Kazakov, A.E.; Arkin, A.P.; Dubchak, I.; Gelfand, M.S. Comparative genomics of the dormancy regulons in mycobacteria. J. Bacteriol. 2011, 193, 3446-3452. [CrossRef]

44. Rodriguez-Sevilla, G.; Garcia-Coca, M.; Romera-Garcia, D.; Aguilera-Correa, J.J.; Mahillo-Fernandez, I.; Esteban, J.; Perez-Jorge, C. Non-Tuberculous Mycobacteria multispecies biofilms in cystic fibrosis: Development of an in vitro Mycobacterium abscessus and Pseudomonas aeruginosa dual species biofilm model. Int. J. Med. Microbiol. 2018, 308, 413-423. [CrossRef] [PubMed]

45. Wayne, L.G.; Hayes, L.G. An in vitro model for sequential study of shiftdown of Mycobacterium tuberculosis through two stages of nonreplicating persistence. Infect. Immun. 1996, 64, 2062-2069. [CrossRef] [PubMed]

46. Carter, G.; Wu, M.; Drummond, D.C.; Bermudez, L.E. Characterization of biofilm formation by clinical isolates of Mycobacterium avium. J. Med. Microbiol. 2003, 52, 747-752. [CrossRef]

47. Greendyke, R.; Byrd, T.F. Differential antibiotic susceptibility of Mycobacterium abscessus variants in biofilms and macrophages compared to that of planktonic bacteria. Antimicrob. Agents Chemother. 2008, 52, 2019-2026. [CrossRef]

48. Ortiz-Perez, A.; Martin-de-Hijas, N.; Alonso-Rodriguez, N.; Molina-Manso, D.; Fernandez-Roblas, R.; Esteban, J. Importance of antibiotic penetration in the antimicrobial resistance of biofilm formed by non-pigmented rapidly growing mycobacteria against amikacin, ciprofloxacin and clarithromycin. Enferm. Infecc. Microbiol. Clin. 2011, 29, 79-84. [CrossRef]

49. Via, L.E.; England, K.; Weiner, D.M.; Schimel, D.; Zimmerman, M.D.; Dayao, E.; Chen, R.Y.; Dodd, L.E.; Richardson, M.; Robbins, K.K.; et al. A sterilizing tuberculosis treatment regimen is associated with faster clearance of bacteria in cavitary lesions in marmosets. Antimicrob. Agents Chemother. 2015, 59, 4181-4189. [CrossRef]

50. Liu, X.; Van Natta, K.; Yeo, H.; Vilenski, O.; Weller, P.E.; Worboys, P.D.; Monshouwer, M. Unbound drug concentration in brain homogenate and cerebral spinal fluid at steady state as a surrogate for unbound concentration in brain interstitial fluid. Drug Metab. Dispos. 2009, 37, 787-793. [CrossRef]

51. Sarathy, J.P.; Zuccotto, F.; Hsinpin, H.; Sandberg, L.; Via, L.E.; Marriner, G.A.; Masquelin, T.; Wyatt, P.; Ray, P.; Dartois, V. Prediction of Drug Penetration in Tuberculosis Lesions. ACS Infect. Dis. 2016, 2, 552-563. [CrossRef] [PubMed]

52. Thimmaiah, K.N.; Easton, J.B.; Germain, G.S.; Morton, C.L.; Kamath, S.; Buolamwini, J.K.; Houghton, P.J. Identification of N10-substituted phenoxazines as potent and specific inhibitors of Akt signaling. J. Biol. Chem. 2005, 280, 31924-31935. [CrossRef]

53. Kuijl, C.; Savage, N.D.; Marsman, M.; Tuin, A.W.; Janssen, L.; Egan, D.A.; Ketema, M.; van den Nieuwendijk, R.; van den Eeden, S.J.; Geluk, A.; et al. Intracellular bacterial growth is controlled by a kinase network around PKB/AKT1. Nature 2007, 450, 725-730. [CrossRef]

54. Crowle, A.J.; Douvas, G.S.; May, M.H. Chlorpromazine: A drug potentially useful for treating mycobacterial infections. Chemotherapy 1992, 38, 410-419. [CrossRef]

55. Amaral, L.; Kristiansen, J.E.; Viveiros, M.; Atouguia, J. Activity of phenothiazines against antibiotic-resistant Mycobacterium tuberculosis: A review supporting further studies that may elucidate the potential use of thioridazine as anti-tuberculosis therapy. J. Antimicrob. Chemother. 2001, 47, 505-511. [CrossRef] [PubMed]

56. Waters, N.J.; Jones, R.; Williams, G.; Sohal, B. Validation of a rapid equilibrium dialysis approach for the measurement of plasma protein binding. J. Pharm. Sci. 2008, 97, 4586-4595. [CrossRef] 
57. Di, L.; Umland, J.P.; Trapa, P.E.; Maurer, T.S. Impact of recovery on fraction unbound using equilibrium dialysis. J. Pharm. Sci. 2012, 101, 1327-1335. [CrossRef] [PubMed]

58. Kalvass, J.C.; Maurer, T.S. Influence of nonspecific brain and plasma binding on CNS exposure: Implications for rational drug discovery. Biopharm. Drug Dispos. 2002, 23, 327-338. [CrossRef] [PubMed] 